\title{
Low-Complexity Compensation of SOA Nonlinearity for Single-Channel PSK and OOK
}

\author{
Francesco Vacondio, Amirhossein Ghazisaeidi, Alberto Bononi, and Leslie Ann Rusch, Senior Member, IEEE
}

\begin{abstract}
Carrier density fluctuations in semiconductor optical amplifiers (SOAs) impose penalties on phase-shift keying (PSK) signals due to nonlinear phase noise (NLPN), and on-off keying (OOK) signals due to self-gain modulation. In this paper, we propose a simple scheme to equalize the impairments induced by SOA nonlinearities, derived from the small signal analysis of carrier density fluctuations. We demonstrate via simulation almost complete cancelation of the NLPN added by a saturated SOA on a differential PSK signal. We demonstrate via both simulations and experiment the effectiveness of the method for mitigation of nonlinear distortions imposed by SOAs on an OOK signal.
\end{abstract}

\section{INTRODUCTION}

$\mathbf{S}$ EMICONDUCTOR OPTICAL AMPLIFIERS are a very interesting alternative to erbium-doped fiber amplifiers (EDFAs) due to their wide spectral gain, compact size, integrability, and cost effectiveness [1]. The nonlinear behavior of semiconductor optical amplifiers (SOAs) can be another attractive feature. For example, SOAs are used as building blocks for $2 R$ regenerators [2], wavelength converters [3], nonlinear media for four-wave mixing [4], or intensity noise suppressors [5]. EDFAs remain the amplifiers of choice when linearity is essential. When SOAs are used strictly as amplifiers, gain variations induce distortions and impose performance penalties. We will discuss how these nonlinearities can be overcome in SOAs.

SOA gain dynamics are determined by carrier recombination and few ultrafast processes (spectral hole burning, carrier heating, Kerr effect, and two photon absorption) [6]. Due to their fast response, these processes become important when working with subpicosecond pulses. In this paper, we focus rather on the gain fluctuations caused by carrier recombination, whose time scale is dominated by the carrier lifetime, typically between a few picoseconds and several hundred picoseconds.

The carriers inside the SOA can be thought as one single reservoir of carriers replenished by the dc current and from which the optical signals drain carriers for amplification [7].

Manuscript received June 11, 2009; revised September 08, 2009. First published November 24, 2009; current version published January 15, 2010. This work was supported by the 2007-2009 Quebec-Italy Executive Program for Scientific Development under Project 13.

F. Vacondio, A. Ghazisaeidi, and L. A. Rusch are with the Center of Optics, Photonics and Laser and the Department of Electrical and Computer Engineering, Université Laval, Quebec, QC G1V0A6, Canada (e-mail: rusch@ulaval.ca).

A. Bononi is with the Dipartimento di Ingegneria dell'Informazione, School of Engineering, Università degli Studi di Parma, 43100 Parma, Italy.

Color versions of one or more of the figures in this paper are available online at http://ieeexplore.ieee.org.

Digital Object Identifier 10.1109/JLT.2009.2036868
The gain is a function of the amount of carriers in the reservoir. When the SOA is saturated, any variation of the input power translates into a variation of the level of the reservoir, which, in turn, the reservoir variations cause variations of the instantaneous gain. For typical SOA carrier lifetimes, the gain can follow the variations of the input power over a bandwidth of the order of gigahertz.

In the case of ON-OFFkeying (OOK), this behavior leads to nonlinear distortions being imposed on the waveform [8]. The problem is less important for very low bit rates, i.e., when the bit time is much smaller than the SOA carrier lifetime $T_{B} \ll \tau_{c}$, or at very high bit rates, i.e., when $T_{B} \gg \tau_{c}$. For typical SOAs, significant bit pattern distortions are imposed on the signals with a bit rate in the range from 1 to $30 \mathrm{~Gb} / \mathrm{s}$.

To overcome this problem, SOAs have been used with differential phase-shift keying (DPSK) [9], quadrature differential phase-shift keying (DQPSK) [10], and other constant or quasi-constant envelope modulation formats [11]. When phase-modulated signals are amplified with SOAs, the limiting nonlinear impairment becomes nonlinear phase noise (NLPN). NLPN arises whenever intensity noise is translated into phase noise through self-phase modulation (SPM). This is a very well known and widely studied phenomenon in optical fiber, named the Gordon-Mollenauer effect [12]. Fiber NLPN is due to the Kerr effect, whereas NLPN in SOAs arises from the refractive index modulation due to carrier density fluctuations [13]. In nonlinear fiber links, the NLPN bandwidth is limited to the nonlinear diffusion bandwidth, inversely proportional to the map strength [14]. In SOAs, on the other hand, the NLPN has the same bandwidth as the gain fluctuations.

NLPN in SOAs was investigated, for example, in [15] and [16]. A good approximation of the relation between the output and the input phases to the SOA [17] is

$$
\varphi_{\text {out }}(t)=\varphi_{\text {in }}(t)+\frac{\alpha}{2} h(t)
$$

where $\alpha$ is the linewidth enhancement factor of the SOA and $h(t)$ is the integrated gain. When the input to the SOA is a constant (or quasi-constant) envelope signal with finite optical SNR (OSNR), its intensity suffers from random fluctuations due to the noise. If the SOA is saturated, these fluctuations modulate the reservoir of carriers and hence the SOA gain $h(t)$. The mechanism by which these fluctuations translate into phase noise is given in (1); note that an SOA with zero linewidth enhancement factor would exhibit no NLPN. The linewidth enhancement factor is an unavoidable, inherent characteristic of SOAs, deriving from changes in the real part of the refractive index 
[18]. In this paper, we consider static $\alpha$ and neglect the complex dynamics of $\alpha$ as they only become important at very high bit rates [19].

The literature is rich with proposals for the compensation of NLPN in fiber [20]-[25]. There are also various studies of compensation of SOA nonlinearities. For instance, SOA bias current can be modulated to counterbalance the reservoir fluctuations by injecting more or less carriers according to the instantaneous input power [26]. The authors in [27] propose an all-optical, linear equalizer made by two Mach-Zehnder interferometers to combat gain variation-induced distortions. The reduction of SOA-induced nonlinear impairments is achieved in [28] with the use of electronic maximum-likelihood sequence estimation at the receiver. A very recent paper [29] proposes digital signal processing that follows coherent detection; the SOA dynamic gain equation is solved numerically, implementing SOA backpropagation in an IC.

In this paper, we use small signal analysis of the SOA dynamic equation to estimate the gain fluctuations and compensate SOA nonlinearity. Our solution is simpler and more cost efficient than those previously proposed. We consider both intensity modulation and differential phase modulation. For phase modulation, we alleviate NLPN via an optoelectronic feedforward mechanism. In the case of intensity modulation, an electrical filter is used to derive a signal to correct for the distortion of the received waveform.

As in [25], for fiber-induced NLPN, we also exploit the correlation between intensity and phase noise due to nonlinear interaction. The novelty of our solution stems from the different nature of NLPN in fiber and SOAs. For SOA-induced NLPN, we can virtually compensate all the phase noise introduced by the SOA with an electrical filter whose optimal characteristic we derive from SOA equations. This is contrary to fiber NLPN; as discussed in [25], postcompensation cannot remove all the fiber NLPN, since the nonlinear phase shift is proportional to the path-averaged intensity, and not the intensity at the end of the link.

In this paper, we focus on single-channel systems, i.e., when SOAs are used as building blocks for monolithically integrated modulators, [30], receivers [31], or other agile network components such as wavelength converters [32], [33]. On the other hand, our solution might be extended to the wavelength-division multiplexed (WDM) scenario, where SOAs would amplify a comb of signals. When more than one channel is present at the SOA input, the reservoir of carriers fluctuates as a function of the total WDM signal power, causing nonlinearities. Given the low-pass nature of the SOA, the reservoir fluctuations have a bandwidth in the order of a few gigahertz. These fluctuations can therefore be estimated and corrected with the schemes we propose. Nevertheless, the extension of our study to the WDM case is not straightforward since gain might vary from channel to channel, depending on the number of channels, their spacing, and the spectral shape of the SOA gain. We leave this matter as a subject of future investigations.

This paper is organized as follows. In the next section, we introduce our proposal for SOA nonlinearity postcompensation based on a small signal model for the SOA. In Section III, we describe a large-signal SOA numerical model that we will use to assess the validity of our NLPN compensation method in Section IV. In Section V, we investigate via simulation and experiment the equalization of distortions induced by the SOA on a $10 \mathrm{~Gb} / \mathrm{s}$ OOK signal. We conclude this paper in Section VI.

\section{EXPLOITING THE LOW-PASS NATURE OF SOAS}

According to the model presented in [17] and [34], the relation between the output and the input fields to the SOA is

$$
E_{\text {out }}(t)=E_{\text {in }}(t) e^{h(t)(1+j \alpha) / 2}
$$

where $\alpha$ is the SOA linewidth enhancement factor and $h(t) \triangleq$ $\int_{0}^{L} g(z, t) d z$ is the material gain integrated over the length of the amplifier. Since $g(t, z)$ is proportional to the carrier density, the quantity $h(t)$ can be thought of as a properly normalized reservoir of carriers available for amplification. As we pointed out in Section I, a major source of nonlinearity is the temporal fluctuations of $h(t)$. The equation governing the dynamics of $h(t)$ is [17], [34]

$$
\tau_{c} \frac{d}{d t} h(t)=h_{0}-h(t)-\left[e^{h(t)}-1\right] p_{\text {in }}(t)
$$

where $h_{0}$ is the unsaturated material gain of the SOA and $p_{\text {in }}(t)$ is defined as the input power normalized to the saturation power of the amplifier: $p_{\text {in }}(t) \triangleq\left|E_{\text {in }}(t)\right|^{2} / P_{\text {sat }}$. In this model, the SOA internal losses are neglected, so that the spatial distribution of the carriers along the propagation direction $z$ do not affect the total gain. It is trivial from (2) and (3) to derive the differential equation relating $h(t)$ and the output power $p_{\text {out }}(t) \triangleq$ $\left|E_{\text {out }}(t)\right|^{2} / P_{\text {sat }}$

$$
\tau_{c} \frac{d}{d t} h(t)=h_{0}-h(t)+\left[e^{-h(t)}-1\right] p_{\text {out }}(t) .
$$

We can now write the gain and the output power as their mean values plus a zero-average term as follows: $h(t)=\bar{h}+\delta h(t)$ and $p_{\text {out }}(t)=\bar{p}_{\text {out }}+\delta p_{\text {out }}(t)$. It is well known that the SOA acts, in the small signal approximation (i.e., when $\delta h(t) \ll \bar{h}$ ), as a low-pass filter between the optical intensity and the gain fluctuations [34]. In the case of output power, we can write

$$
\delta h_{l}(t) \simeq K \delta p_{\text {out }}(t) \otimes m(t)
$$

where the subscript $l$ reminds us that a linearization of (4) was performed, $\otimes$ denotes the convolution, $K$ is

$$
K=\frac{e^{-\bar{h}}-1}{1+\bar{p}_{\text {out }} e^{-\bar{h}}}
$$

and $m(t)$ is a single-pole low-pass filter with time constant

$$
\tau_{\text {eff }}=\frac{\tau_{c}}{1+\bar{p}_{\text {out }} e^{-\bar{h}}} .
$$

In Fig. 1, we report a contour plot of the $3 \mathrm{~dB}$ bandwidth of the filter as a function of the SOA carrier lifetime $\tau_{c}$ and the input average power $\bar{p}_{\text {in }}$. The $3 \mathrm{~dB}$ bandwidth is smaller than a few gigahertz for a wide range of realistic values of input power, when the carrier lifetime is larger than $50 \mathrm{ps}$. The carrier lifetime $\tau_{c}$, an intrinsic parameter of every SOA, is swept in the wide range 


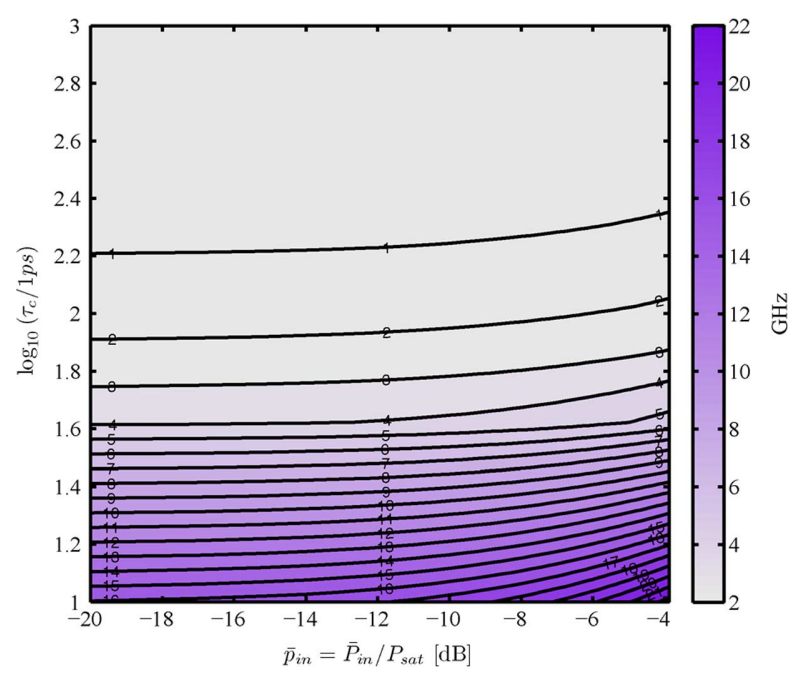

Fig. 1. $3 \mathrm{~dB}$ bandwidth of $M(\omega)$ as a function of the two parameters that completely determine its time constant.

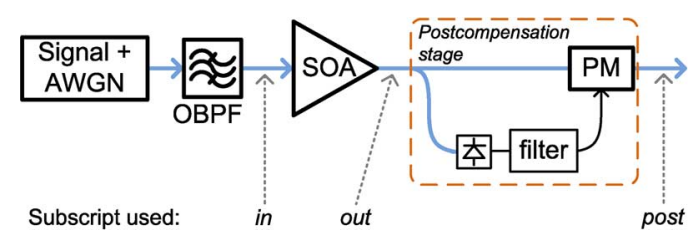

Fig. 2. Signal (either $\mathrm{CW}$ or phase-modulated DPSK) plus additive white Gaussian noise (AWGN) pass through an optical bandpass filter (OBPF) before entering an SOA. The postcompensation stage includes a photodiode, an electrical filter, and a PM.

of $10 \mathrm{ps}$ to $1 \mathrm{~ns}$. At carrier lifetimes smaller than approximately $20 \mathrm{ps}$, the bandwidth of the filter becomes as large as $10 \mathrm{GHz}$.

In this paper, we will exploit (5) to estimate the gain variations by observing the output power. We will then compensate for the SOA nonlinearities both for phase-modulated and intensity-modulated signals. We begin with phase modulation and compensate the SOA-induced nonlinear phase noise. The reference link is outlined in Fig. 2. The input signal (either a continuous-wave $(\mathrm{CW})$ laser or a phase-modulated signal) is optically filtered and injected in an SOA. After amplification, there is a compensation stage composed of an ac-coupled photodiode, an electrical filter, and a phase modulator (PM). The optimal bandwidth of the electrical filter depends on the saturation level of the SOA and also on the average optical input power (see, e.g., Fig. 1). The average input power to the line amplifiers is an important design parameter of an optical network, and it should not vary much with time. Nevertheless, simple low-frequency circuits can be devised to monitor the input average power and adjust the filter bandwidth accordingly.

As can be seen in Fig. 2, and using (1), the phase after postcompensation can be written as

$$
\begin{aligned}
\varphi_{\text {post }}(t) & =\varphi_{\text {out }}(t)+C \delta p_{\text {out }}(t) \otimes m(t) \\
& =\varphi_{\text {in }}(t)+\frac{\alpha}{2} h(t)+C \delta p_{\text {out }}(t) \otimes m(t)
\end{aligned}
$$

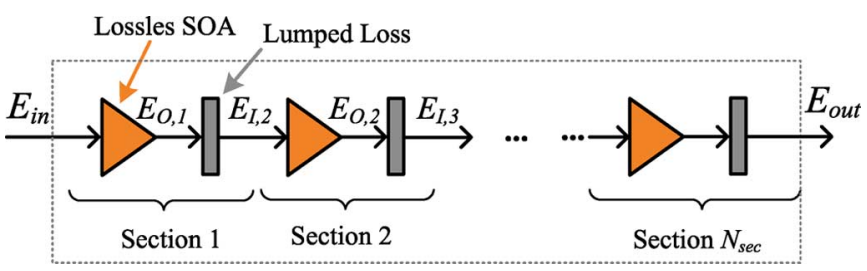

Fig. 3. SOA model includes $N_{\mathrm{sec}}$ sections along the propagation direction, each with a lossless "short" SOA and a lumped loss.

where we have taken the SOA low-pass filter $m(t)$ impulse response and implemented it in an electrical filter. The constant $C$ is used to take into account the effect of splitting loss, photodiode responsivity, filter losses, and PM efficiency.

By writing $h(t)=\bar{h}+\delta h(t)$ and using (5), we can reformulate the postcompensated phase as

$$
\begin{aligned}
\varphi_{\text {post }}(t) & =\varphi_{\text {in }}(t)+\frac{\alpha}{2} \bar{h}+\frac{\alpha}{2} \delta h(t)+C \delta h_{l}(t) / K \\
& \simeq \varphi_{\text {in }}(t)+\frac{\alpha}{2} \bar{h}
\end{aligned}
$$

where, in the last passage, we choose $C=-K \alpha / 2$. Thus, under the small signal assumption, we can perfectly suppress the NLPN introduced by the SOA, leaving only an offset to the input phase. We turn to numerical simulation to confirm our analysis and quantify the validity of the small signal assumption.

\section{LARGE- Versus SMALL-SignAl ModelS}

Here, we introduce the large-signal SOA model that we will use in the simulations reported throughout the paper. We use the space-resolved model described in [35] that captures the distributed nature of the carrier density along the propagation direction $z$, as well as the distributed intrinsic losses of the SOA. Note that neglecting these features was the main drawback of the small-signal analysis of the previous section.

The schematic of the model is outlined in Fig. 3. An SOA of length $L$ is divided into $N_{\mathrm{sec}}$ sections. We used $N_{\mathrm{sec}}=10$ as results were unchanged with larger $N_{\text {sec }}$. Each section is composed of a lossless SOA of length $L / N_{\mathrm{sec}}$ followed by a lumped loss. The equations governing the $i$ th section are

$$
\begin{aligned}
E_{O, i}(t) & =E_{I, i}(t) e^{h_{i}(t)(1+j \alpha) / 2} \\
\tau_{c} \frac{d}{d t} h_{i}(t) & =h_{0}-h_{i}(t)-\left[e^{h_{i}(t)}-1\right] \frac{\left|E_{I, i}\right|^{2}}{P_{\text {sat }}}
\end{aligned}
$$

where $E_{I, i}(t)$ and $E_{O, i}(t)$ are the input and output fields of the $i$ th section, respectively. The unsaturated integrated gain of each SOA section is $h_{0}$ and $h_{i}(t)$ is the integrated gain of the $i$ th section. The initial condition is $E_{I, 1}(t)=E_{\mathrm{in}}(t)$, and for each section, after the first the input field is the attenuated output of the previous section per

$$
E_{I, i}(t)=E_{O, i-1}(t) e^{\left(-\alpha_{\mathrm{int}} L / N_{\mathrm{sec}}\right)}, \quad i \geq 2 .
$$

The output field is therefore $E_{\text {out }}(t)=E_{I, N_{\mathrm{sec}}+1}(t)$. 


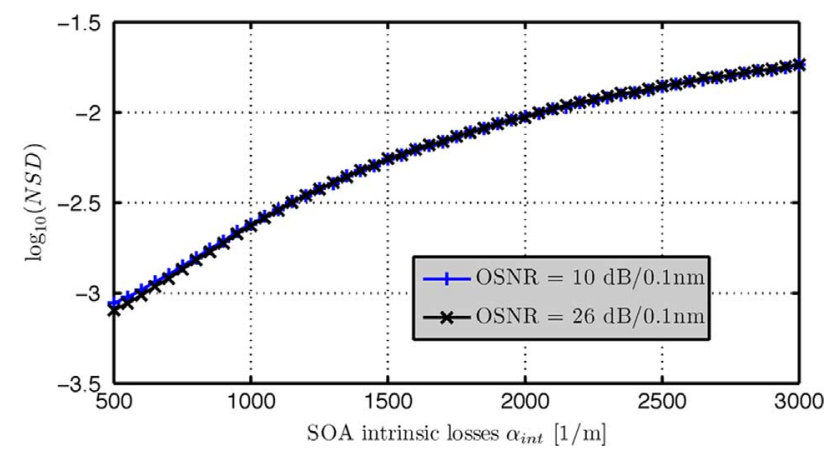

Fig. 4. Logarithmic plot of the NSD as function of the SOA intrinsic losses.

In our simulations, we neglect internal amplified spontaneous emission (ASE). In [35], the ASE is treated as a white noise added to the input signal. Hence, including the ASE would translate into a different OSNR at the SOA input. We will show that the accuracy of the proposed method shows very little dependence on the input OSNR, and therefore, results would not change when including ASE as in [35].

The total integrated gain $h_{T}(t)$, as distinguished from the lossless gain $h(t)$ of the single-reservoir model of the previous section, is defined as

$$
h_{T}(t)=\ln \left(p_{\text {out }} / p_{\text {in }}\right)=\sum_{i=1}^{N_{\text {sec }}} h_{i}(t)-\alpha_{\text {int }} L .
$$

The fluctuation, not necessarily small, of the gain around its average is $\delta h_{T}(t) \triangleq h_{T}(t)-\left\langle h_{T}(t)\right\rangle$. In order to establish the effectiveness of (5), we compare the "true" results of the large-signal model $\delta h_{T}(t)$ against the result of the small-signal approximation (5) in terms of the normalized standard deviation (NSD)

$$
\mathrm{NSD}=\frac{\left|\int_{-\infty}^{+\infty}\left[\delta h_{T}(t)-\delta h_{l}(t)\right]\right|^{2}}{\left|\int_{-\infty}^{+\infty} \delta h_{T}(t)\right|^{2}} .
$$

The input signal to the SOA (parameters in Table I) is a CW laser plus additive white Gaussian noise (AWGN) filtered by a second-order super-Gaussian filter with a $3 \mathrm{~dB}$ bandwidth of $15 \mathrm{GHz}$. SOA parameters were chosen to give realistic results when compared with experimental data [36], [37]. The total signal power is $3 \mathrm{dBm}$, and two disparate OSNRs are considered, 10 and $26 \mathrm{~dB}$. Fig. 4 shows the NSD calculated from (14), where $\delta h_{T}(t)$ is obtained numerically with the large-signal SOA model and $\delta h_{l}(t)$ is obtained from (5). We sweep a wide range of intrinsic loss $\alpha_{\text {int }}$; NSD is always below $1.8 \times 10^{-2}$, regardless of the signal OSNR. To visualize the accuracy of the small-signal model, we plot in Fig. 5 a portion of the waveforms for OSNR $=10 \mathrm{~dB}$ and $\alpha_{\text {int }}=3000$.

For a CW laser corrupted by filtered AWGN, the power spectral densities (PSDs) at SOA input and output are shown in Fig. 6(a) and (b), respectively, for phase and intensity [13]. In Fig. 6(b), we show the advantageous reduction in intensity noise at low frequencies. In Fig. 6(a), we have instead an enhancement of phase noise at low frequencies, a manifestation of the

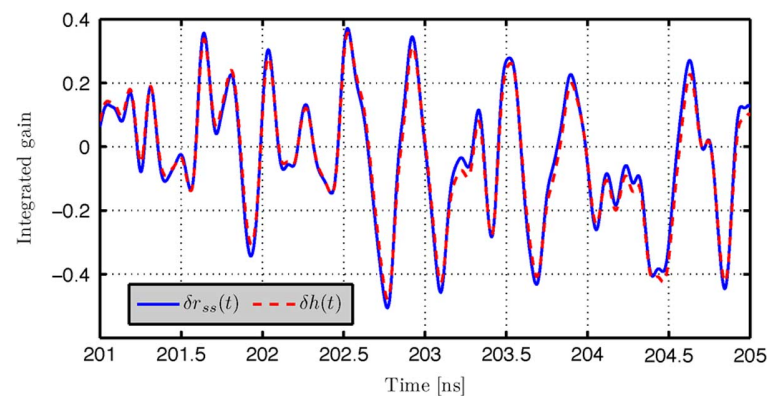

Fig. 5. Predictions of integrated gain from large $\delta h_{T}(t)$ versus small $\delta h_{l}(t)$ signal models; NSD is 0.018 in this example.

TABLE I

PARAMETERS USED IN THE SimUlation (UNLeSS SPECIFIED OTHERWISE IN THE TEXT)

\begin{tabular}{|c||c||c|}
\hline Parameter & Value & Units \\
\hline SOA length $L$ & 650 & $\mu \mathrm{m}$ \\
\hline$\alpha$ & 7 & \\
\hline$\tau_{c}$ & 175 & $\mathrm{ps}$ \\
\hline$h_{0}$ & 6.22 & \\
\hline$P_{\text {sat }}$ & 13 & $\mathrm{dBm}$ \\
\hline Intrinsic losses $\alpha_{\text {int }}$ & 2180 & $1 / \mathrm{m}$ \\
\hline Number of sections & 10 & \\
\hline \multicolumn{2}{|c}{} \\
\hline
\end{tabular}
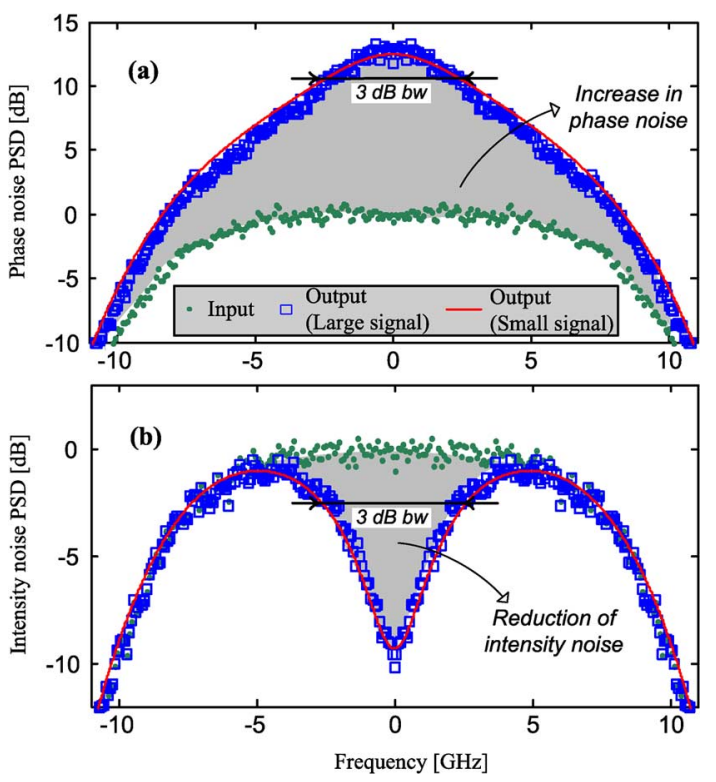

Fig. 6. PSD of intensity and phase noise at the input and output of the SOA. The input signal is a CW laser plus AWGN. OSNR is $23 \mathrm{~dB}$, noise is filtered with a super-Gaussian filter of order 2, with $15 \mathrm{GHz} 3 \mathrm{~dB}$ bandwidth.

NLPN problem. The SOA gives an advantage in intensity noise suppression that we wish to maintain, but an enhancement of phase noise that we wish to combat. Predictions from large- and small-signal models coincide.

The bandwidth of the noise redistribution (a few gigahertz) indicates the speed with which the reservoir can follow the input power fluctuations. The novel, important message of Fig. 6 is that numerical PSD calculated with the large-signal model and the small-signal PSD are very similar, validating our exploitation of small-signal analysis of the single-reservoir model that neglects SOA intrinsic losses. 


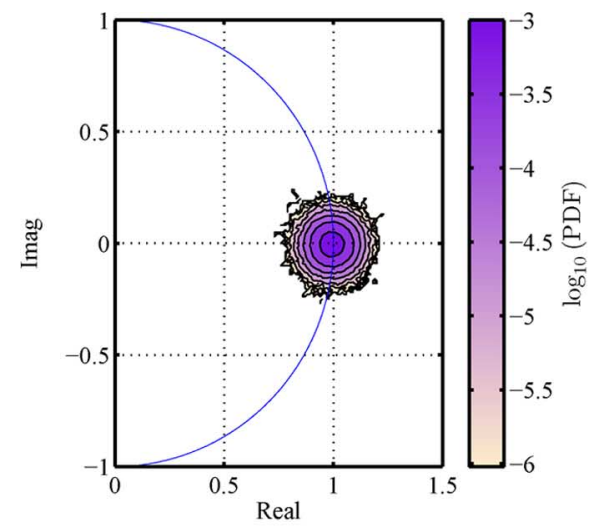

(a)

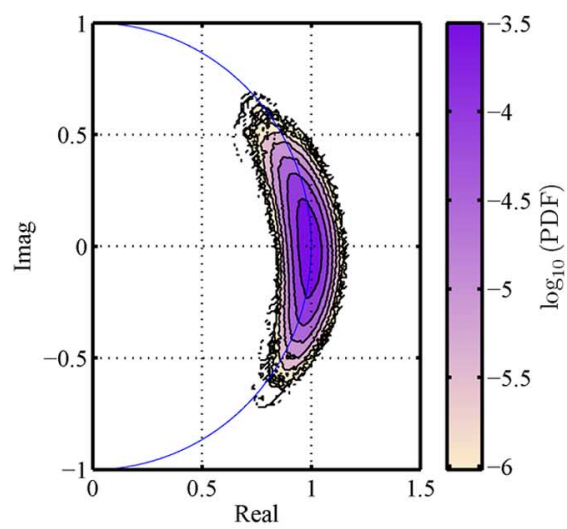

(b)

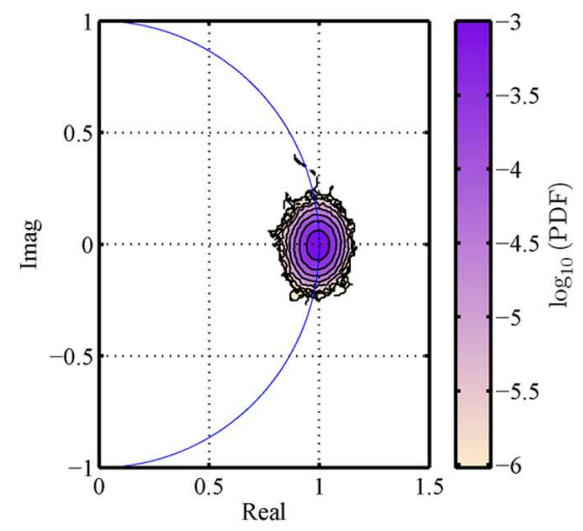

(c)

Fig. 7. Logarithmic 2-D PDF of the complex electric fields; input power is $0 \mathrm{dBm}$, OSNR $=23 \mathrm{~dB}$, super-Gaussian optical filter of order 2 and $15 \mathrm{GHz}$ bandwidth.

\section{NLPN COMPENSATION}

In this section, we will numerically implement the scheme shown in Fig. 2 and evaluate its effectiveness. For the sake of generality, and in order to clarify the principle of the compensator, we will begin with the simple case where the signal is a noisy $\mathrm{CW}$ laser and evaluate the phase noise variance suppression. The proposed method would work with any phase-modulated scheme; we will show as an example the case of binary DPSK modulation and calculate the noise suppression in terms of differential phase $Q$ factor.

\section{A. NPLN Reduction: Noisy CW Laser}

Consider a simple CW laser with filtered additive Gaussian noise, filtered by a super-Gaussian optical filter (order 2 and 15 $\mathrm{GHz} 3 \mathrm{~dB}$ bandwidth), and injected into an SOA. The SOA is simulated with the large-signal model of the previous section, with parameters listed in Table I. The nonlinear phase noise generated by the SOA is compensated with the scheme shown in Fig. 2.

In this first round of simulations, the input power to the SOA is fixed at $0 \mathrm{dBm}$. In Fig. 7(a), we described the 2-D probability density function (PDF) of the electric field in the complex plane at the input of the SOA, where the Gaussian noise gives a bell-shaped PDF in two dimensions (circular contours). Fig. 7(b) is the PDF of the signal at the output of the SOA. Any constant phase shift has been ignored, and all PDFs are centered at $(1,0)$. As predicted by the PSDs shown in Fig. 6, we can see a clear enhancement of the phase noise and a reduction (squeezing) of the intensity noise. Note that the real and imaginary parts of the electric fields are now strongly correlated due to the NLPN process. Finally, Fig. 7(c) shows the PDF of the signal at the output of the postcompensation stage described in Fig. 2 when using the optimal $C$ in (8). The phase noise has clearly been reduced, whereas the intensity noise remains suppressed. In other words, this compensator preserves the reduction in the intensity noise, and drastically reduces the phase noise introduced by the SOA. The net effect is that of having an SOA with almost zero linewidth enhancement factor, i.e., no NLPN.

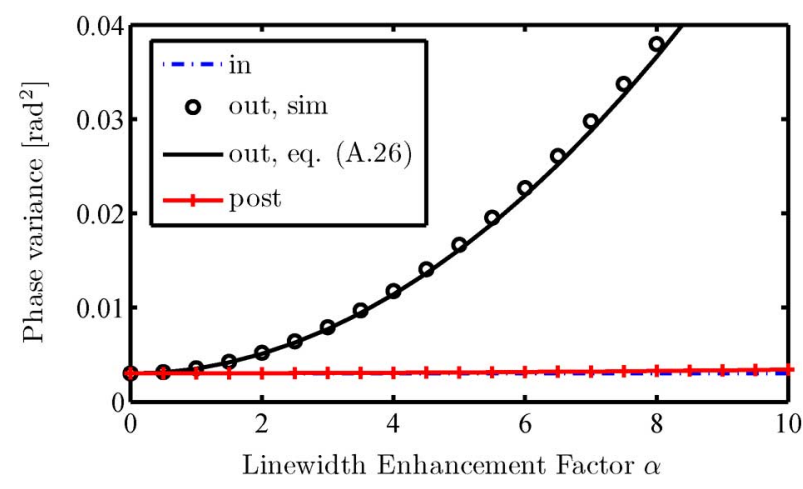

Fig. 8. Phase variance versus linewidth enhancement factor, $P_{\text {in }}=0 \mathrm{dBm}$.

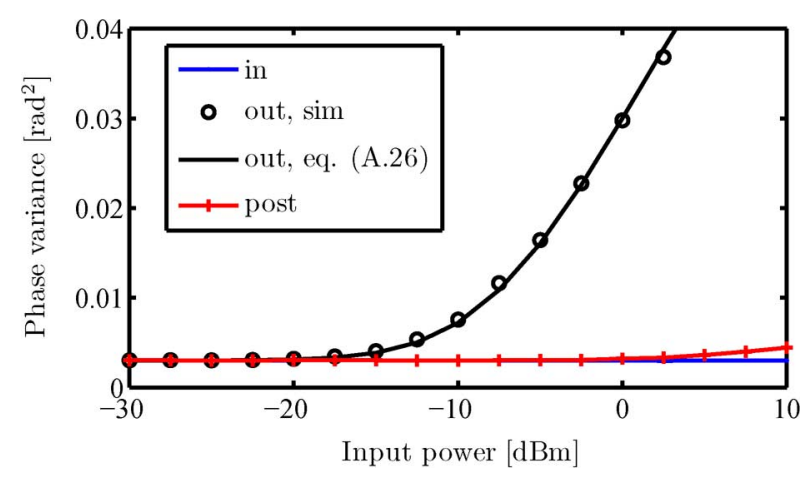

Fig. 9. Phase variance versus SOA input power, $\alpha=7$.

To better quantify our results, we figure in Fig. 8 the variance of the optical phase versus SOA linewidth enhancement factor $\alpha$ at the three test points. The postcompensation stage is very effective, almost perfectly compensating the NLPN added by the SOA. The discrepancy between the small-signal approximation and the true signal is acerbated by $\alpha$ per (9); compensation is expected to be less accurate for very high values of $\alpha$. Nonetheless, for realistic values of $\alpha$ shown in Fig. 8, the discrepancy is barely notable.

We also expect the small-signal approximation (and therefore the compensator itself) to be less accurate when working in deep saturation. To investigate this case, we sweep the SOA input 


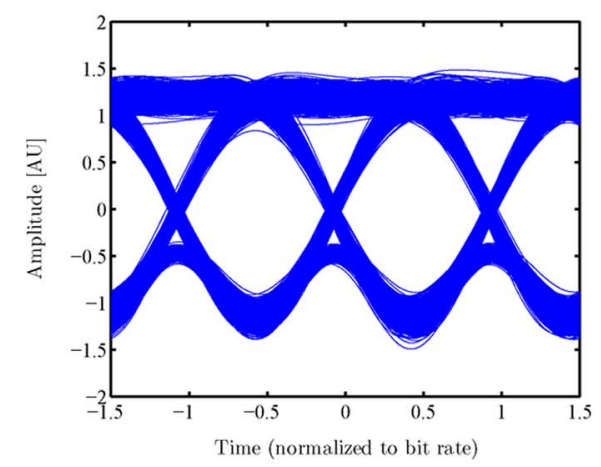

(a)

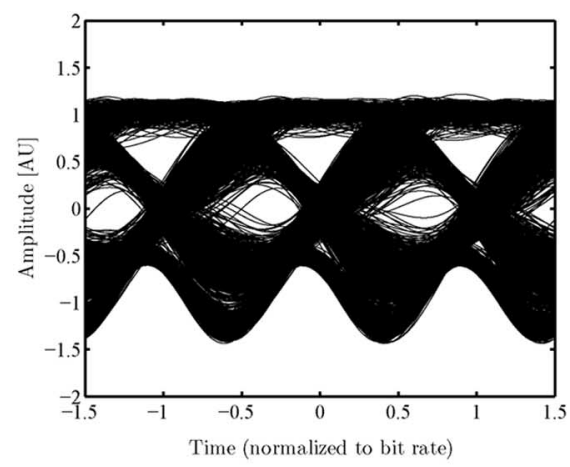

(b)

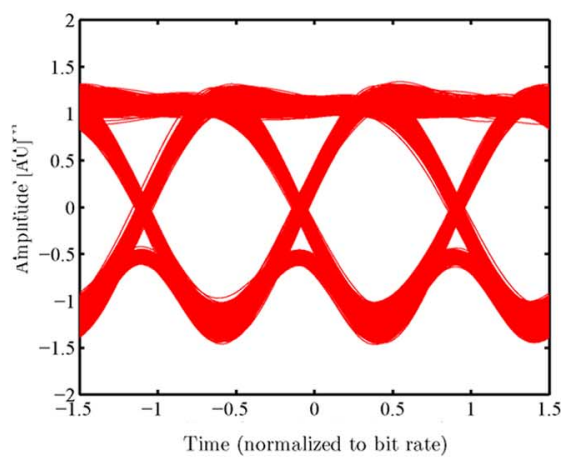

(c)

Fig. 10. DPSK eye diagrams after demodulation; input power is $0 \mathrm{dBm}$, OSNR $=23 \mathrm{~dB}$, super-Gaussian optical filter of order 2 and $15 \mathrm{GHz}$ bandwidth. (a) Input to SOA. (b) Output. (c) Postcompensated.

power up to $+10 \mathrm{dBm}$, with resulting phase noise variances at the input, output, and following postcompensation shown in Fig. 9. The method is very effective even at very high saturation levels: the gain compression at $+10 \mathrm{dBm}$ of input power is $\sim 15 \mathrm{~dB}$.

In the Appendix, we give an analytic expression for the phase noise variance at the SOA output. The calculation is straightforward and a similar one was performed in [16]. It is useful as it shows the quadratic dependence of the output phase noise on $\alpha$ and input power. Moreover, in Figs. 8 and 9, we show that this calculation also agrees with the large-signal model prediction.

\section{B. NPLN Reduction: DPSK Modulation}

Our study of the CW input signal allowed us to unambiguously interpret input/output behavior of the SOA and our compensator; however, this is not the situation where NPLN is problematic. In this section, we show the effect of NLPN postcompensation on the more realistic case of a binary DPSK signal. The signal is generated by an ideal non-return-to zero (NRZ) DPSK transmitter at a bit rate of $R=10 \mathrm{~Gb} / \mathrm{s}$. We use De Brujin sequences of length $2^{13}$ for the bit pattern. The signal is loaded with AWGN, and then optically filtered to limit out-of-band ASE. Simulation parameters are unchanged from the previous section. The scheme for NLPN postcompensation is again the one shown in Fig. 2. The DPSK signal is demodulated incoherently with an ideal 1-bit delay interferometer followed by a balanced detector. In the electronic domain, the photocurrent is then filtered with a fifth-order Bessel-Thompson filter with a $3 \mathrm{~dB}$ bandwidth of $0.65 R$.

Fig. 10 shows the eye diagrams of the received signal after demodulation at the three test points (before the SOA, after amplification, and after postcompensation). The eye diagram after the SOA is closed due to excess phase noise; the eye becomes very open after postcompensation. The eye is actually better after postcompensation than at the SOA input due to the intensity noise reduction introduced by the SOA.

One of the major differences of SOA postcompensation with respect to compensation of fiber-generated NLPN [25] is the role of the electrical filter. We have shown in Section II that an $R C$ filter with time constant $\tau_{\text {eff }}$ completely removes SOA
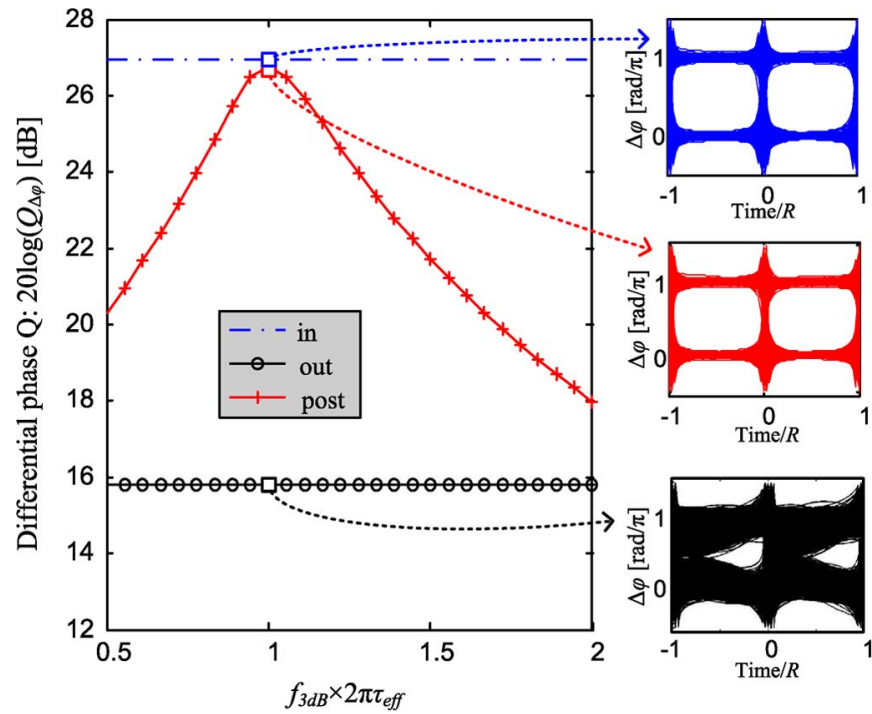

Fig. 11. (Left) Differential phase $\mathrm{Q}$ in decibels versus $m(t) 3 \mathrm{~dB}$ bandwidth; abscissa are normalized to the optimal value derived in the previous section. (Right) Eye diagrams of the differential phase at $f_{3 \mathrm{~dB}}=1 /\left(2 \pi \tau_{\text {eff }}\right)$. The input power is $0 \mathrm{dBm}$, OSNR $23 \mathrm{~dB}$, and optical filter bandwidth $15 \mathrm{GHz}$.

NLPN in the small-signal regime. We now investigate the importance of the bandwidth of postcompensation electrical filter. The differential phase is defined as $\Delta \varphi(t) \triangleq \varphi(t)-\varphi\left(t-T_{B}\right)$, where $T_{B}$ is the bit duration and $\varphi(t)$ is the instantaneous phase of the optical field. We define the differential phase $Q$ factor [38] as

$$
Q_{\Delta \varphi} \triangleq \frac{\pi}{\left(\sigma_{\Delta \varphi_{0}}+\sigma_{\Delta \varphi_{\pi}}\right)}
$$

where $\sigma_{\Delta \varphi_{0}}$ and $\sigma_{\Delta \varphi_{\pi}}$ are the standard deviations of the sampled differential phase on the 0 and $\pi$ rails, respectively. The curve shown in Fig. 11 (left) shows the dependence of $Q_{\Delta \varphi}$ on the bandwidth of the electrical filter. The input power to the SOA is $0 \mathrm{dBm}$, the OSNR is $23 \mathrm{~dB}$, and the $3 \mathrm{~dB}$ bandwidth of the second-order super-Gaussian filter is $15 \mathrm{GHz}$. The $x$-axis is the $3 \mathrm{~dB}$ bandwidth normalized to the optimal value derived in Section II. To be consistent with [25], the differential phase $Q$ is expressed in decibels, i.e., $20 \log Q_{\Delta \varphi}$. With this convention, the $Q$ required for error-free transmission is $\sim 15.6 \mathrm{~dB}$. 


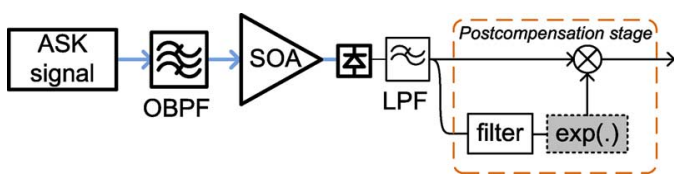

Fig. 12. OOK postcompensation.

In Fig. 11 (right), we present differential phase eye diagrams, where the differential phase is traced in the $[-\pi / 2,3 \pi / 2]$ interval. The eye diagrams are reported for the optimal value of filter bandwidth. The figure demonstrates that, as we expected from the results of the previous section, the postcompensation is very effective in suppressing the NLPN introduced by the SOA, even when the differential phase $Q$ is degraded by as much as $\sim 11 \mathrm{~dB}$ before compensation. Moreover, we see a clear maxima at the optimal filter bandwidth. For this specific case, $Q_{\Delta \varphi}$ after postcompensation is within $1 \mathrm{~dB}$ of the unimpaired $Q$ over a bandwidth range of $200 \mathrm{MHz}$ (from $0.9 \times 2 \pi \tau_{\text {eff }}$ to $1.1 \times 2 \pi \tau_{\text {eff }}$ ), attesting the robustness of this solution.

\section{NONLINEAR DISTORTION COMPENSATION FOR INTENSITY-MODULATED SIGNALS}

We can also exploit the correlation between the SOA output and the gain perturbation induced by NLPN to develop a compensation strategy for intensity-modulated signals. Consider the setup shown in Fig. 12, where the SOA output is again filtered; in this case, the control signal multiplies the output, essentially inverting the gain perturbations. We will evaluate the importance of including or not the exponential function inside the gray dotted box. Without the exponential function, the setup can be built by analog microwave devices (one splitter, one filter, and one mixer).

The postcompensated intensity $p_{\text {post }}$ is given by

$$
\begin{aligned}
p_{\text {post }}(t) & =p_{\text {out }}(t) e^{\left[C \delta h_{l}(t)\right]} \\
& =p_{\text {in }}(t) e^{\left[\bar{h}+\delta h(t)+C \delta h_{l}(t)\right]} \\
& \simeq p_{\text {in }}(t) e^{[\bar{h}]}
\end{aligned}
$$

where $C$ is a properly chosen constant, and we ignored the effect of the low-pass filter LPF for the sake of clarity. The filter will be included in the simulations. Since the input signal to the SOA is an intensity-modulated signal, possibly with high extinction ratio, in principle, $\delta h_{l}(t)$ differs from the true gain $\delta h(t)$, as (5) is the result of a small-signal approximation. As we will see via a numerical example and an experimental validation, the method nonetheless yields very good results.

Note the method as it is cannot compensate for the chirp induced by the SOA on an intensity-modulated signal. This is not an issue if the SOA is used as a preamplifier since photodetection is not sensitive to chirp. It could be a problem if SOAs are used before fiber transmission. SOA-induced chirp could then be compensated with the scheme shown in Fig. 2. Chirp- and intensity-induced distortions could actually be compensated by cascading the two proposed schemes, or even at the same time with the scheme shown in Fig. 2 by substituting the PM with a dual-drive Mach-Zehnder modulator (DDMZM), and deriving

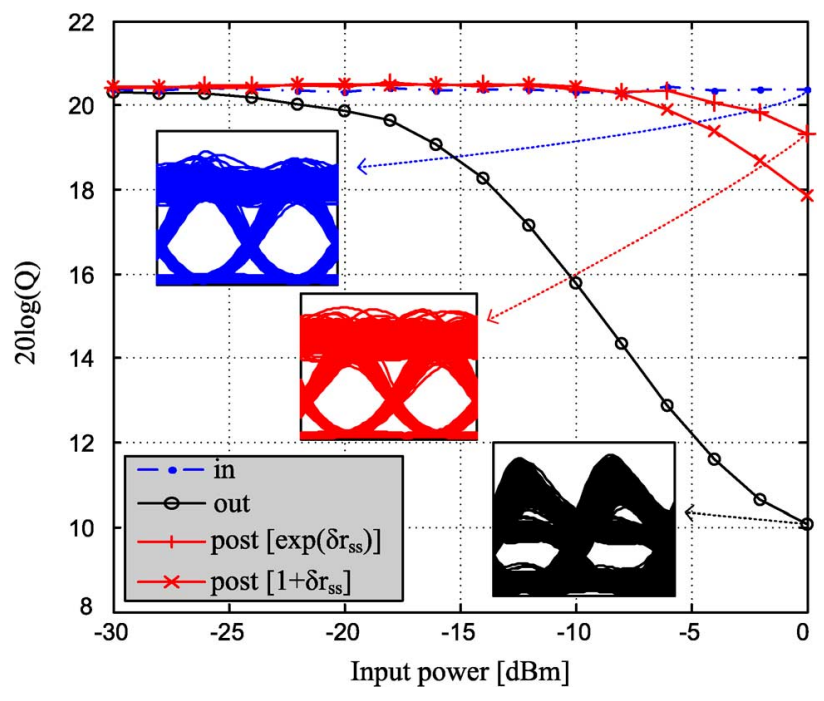

Fig. 13. OOK $Q$ factor at SOA input, output, and after postcompensation.

appropriate filters for the two arms of the DDMZM. We leave this matter for future investigations.

\section{A. Dependence on Saturation Level}

Once again, we expect the small-signal approximation to be less effective when the depth of gain saturation is increased. In this section, we show through a numerical example that the proposed method shown in Fig. 12 works very well up to high values of gain compression. The simulated OOK signal has an extinction ratio of $13 \mathrm{~dB}$ at a bit rate of $R=10 \mathrm{~Gb} / \mathrm{s}$. Noise is added such that its OSNR is $23 \mathrm{~dB}$ over the typical $0.1 \mathrm{~nm}$ bandwidth. Even though in this section we are mainly interested in the deterministic distortions induced on the modulated waveform, we include the noise (with a rather high OSNR) in order to take into account realistic signal-noise interactions inside the SOA also. The noisy signal is then filtered with a second-order super-Gaussian filter with a $3 \mathrm{~dB}$ bandwidth equal to $1.5 R$. The SOA nonlinear gain fluctuations distort the signal. The SOA output is photodetected and low-pass filtered with a fifth-order Bessel-Thompson filter with $3 \mathrm{~dB}$ bandwidth equal to $R$, in order to emulate a bandwidth-limited detector. The postcompensation stage follows, composed of a splitter, the same $R C$ filter presented in the previous sections with impulse response $m(t)$, and a mixer. The output of the $R C$ filter is an estimate of the integrated gain $\delta h_{l}(t)$, therefore we should apply an exponential function. We explore suppressing the exponential function, allowing implementation in analog microwave devices (splitter, filter, and mixer). In place of the exponential $e^{\delta h_{l}(t)}$, we use its linearized counterpart $\left[1+\delta h_{l}(t)\right]$.

Fig. 13 illustrates the behavior of the simulated $Q$ factor as a function of the average input power to the SOA. As can be seen, the method is very effective up to high input powers. Fig. 13 (insets) shows the eye diagrams at the SOA input, output, and after postcompensation for $0 \mathrm{dBm}$ input power, thereby demonstrating the effectiveness of the method. The maximum gain in $Q$ factor is $\sim 9 \mathrm{~dB}$. Without the exponential block, the postcompensator performance deteriorates slightly, and the maximum gain in $Q$ factor is $\sim 8 \mathrm{~dB}$. 


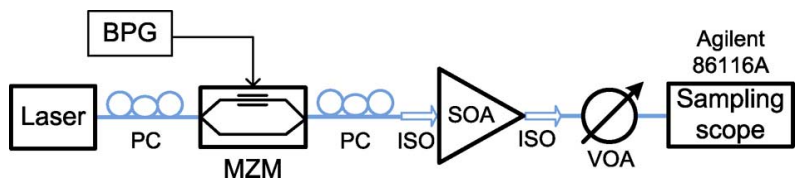

Fig. 14. Experimental setup for OOK postcompensation.
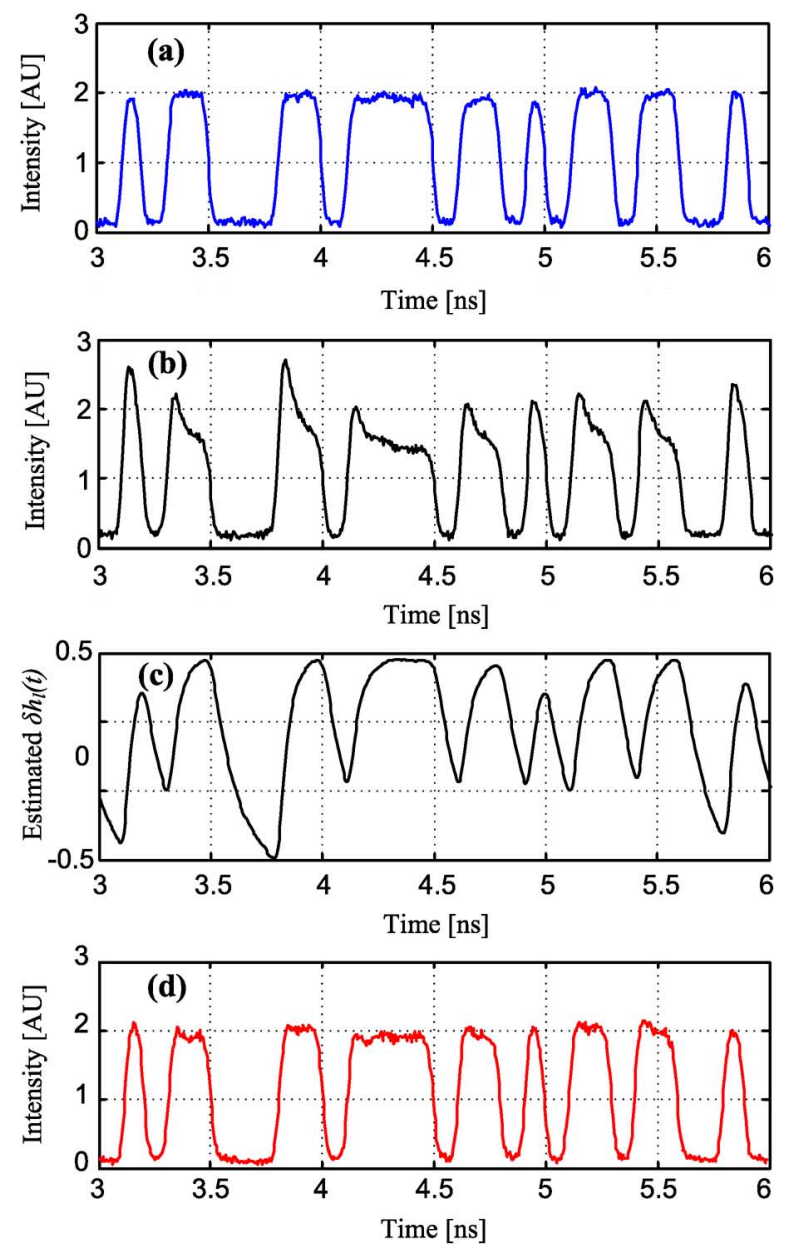

Fig. 15. Measured waveforms of OOK signal at the SOA input (a) and output (b). The result of offline processing are also reported: (c) is $h_{l}(t)$, and (d) is the signal after post-compensation.

\section{B. Experimental Validation}

In order to further establish the effectiveness of the method, we present measured SOA output waveforms, rather than simulated. The experimental setup is outlined in Fig. 14. A polarization-controlled DFB laser is modulated by an MZM driven with a length $2^{7}$ De Brujin sequence. No significant difference in the signal statistics was observed when using a longer sequence. The modulated signal passes another polarization controller (PC) and an isolator (ISO) before entering the SOA with average input power of $-2.65 \mathrm{dBm}$. The SOA output is again isolated to avoid backreflections, and a variable optical attenuator (VOA) is used to control the power on the photodiode. An Agilent 86116A wideband sampling oscilloscope captures the output, with the averaging function invoked; the waveform is the average of 100 realizations. This minimizes the impact of random noise, as we are mostly interested in the deterministic distortions induced by the SOA.

Our simulator was extensively verified to assure good predictions of measured waveforms, as we have reported in [36] and
[37]. The parameters we used are the same as for the numerical predictions of the previous sections, and are presented in Table I. The resulting optimal bandwidth of the low-pass filter is smaller than $1 \mathrm{GHz}$. Fig. 15 summarizes the results of the experiment. Fig. 15(a) shows the measured waveform at the output of the MZM (input to SOA), whereas Fig. 15(b) is the waveform at the output of the SOA. With this signal, we can estimate (offline) $h_{l}(t)$ from (5). The result is shown in Fig. 15(c). Using the scheme proposed in Fig. 12, we obtain the waveform shown in Fig. 15(d), where the distortions introduced by the SOA are almost perfectly canceled.

\section{CONCLUSION}

Starting from the small-signal analysis of the SOA gain dynamic equation, we have presented a simple yet effective method for the postcompensation of SOA-induced nonlinearities. We have shown via numerical simulations, the performance of the nonlinear phase noise compensation for phase-modulated signals, and investigated the range of saturation level and other SOA parameters covered. We have demonstrated both via simulation and experiment its effectiveness for the compensation of SOA-induced waveform distortions on OOK signals. The postcompensation stage shown in Fig. 2 could be integrated into one device, where the new component is the equivalent of a zero linewidth enhancement factor SOA for phase modulation. The postcompensator shown in Fig. 14 could be exploited at the receiver side, alleviating the restrictions imposed by SOAs to the use of intensity-modulated signals.

\section{APPENDIX \\ PHASE NOISE VARIANCE AT SOA OUTPUT}

We analytically calculate the variance of the phase noise at the SOA output $\varphi_{\text {out }}(t)$, under the small-signal assumption when the input field to the SOA is a CW laser plus filtered AWGN, as per Fig. 2. The input field can be written as

$$
E_{\text {in }}(t)=\sqrt{\bar{P}_{\text {in }}}\left[1+\xi\left(n_{p}(t)+j n_{q}(t)\right)\right]
$$

where $j$ is the imaginary unit and $\xi$ is defined as

$$
\xi \triangleq 10^{-0.05 \text { OSNR }} / \sqrt{2}
$$

with OSNR being the signal optical SNR on the typical resolution bandwidth $\Delta_{\mathrm{RB}}=0.1 \mathrm{~nm}$. The real and imaginary parts of the zero-mean Gaussian random noise are $n_{p}(t)$ and $n_{q}(t)$, with equal PSD) given by

$$
S_{n_{p, q}}(\omega)=\frac{1}{\Delta_{\mathrm{RB}}}\left|B_{o}(\omega)\right|^{2}
$$

where $B_{o}(\omega)$ is the frequency response of the optical filter.

We will calculate the variance of the output phase as

$$
\operatorname{var}\left[\varphi_{\text {out }}(t)\right]=\left\langle\varphi_{\text {out }}^{2}(t)\right\rangle-\left\langle\varphi_{\text {out }}(t)\right\rangle^{2}
$$


The average of the output phase is straightforward from (1)

$$
\left\langle\varphi_{\text {out }}(t)\right\rangle^{2}=\left(\frac{\alpha}{2} \bar{h}\right)^{2}
$$

The second moment can be written as

$$
\left\langle\varphi_{\text {out }}^{2}(t)\right\rangle=\xi^{2}\left[\sigma^{2}+\alpha^{2} \bar{p}_{\text {in }}^{2} K^{2} \mu(0)\right]+\left(\frac{\alpha}{2} \bar{h}\right)^{2}
$$

where $\sigma^{2}$ is the variance of $n_{p}(t)$ [equal variance for $n_{q}(t)$ ] and $\mu(t)$ gives the filtering effect of the SOA, defined as

$$
\mu(t) \triangleq m(-t) \otimes m(t) \otimes R_{n_{p, q}}(t)
$$

with $R_{n_{p, q}}(t)$ being the autocorrelation function of $n_{p}(t)$ and $n_{q}(t)$, equal to the inverse Fourier transform of (19) due to the Wiener-Khinchin relation. At $t=0$, we have

$$
\mu(0)=\frac{1}{\Delta_{\mathrm{RB}}} \int_{-\infty}^{\infty} \frac{\left|B_{o}(\omega)\right|^{2}}{1+\omega^{2} \tau_{\mathrm{eff}}^{2}} d \omega
$$

Noting that the variance of the input phase can be written (in the small-signal regime) as

$$
\operatorname{var}\left[\varphi_{\text {in }}(t)\right]=\xi^{2} \sigma^{2}
$$

the output variance can be written as

$$
\operatorname{var}\left[\varphi_{\text {out }}(t)\right]=\operatorname{var}\left[\varphi_{\text {in }}(t)\right]+\alpha^{2} \xi^{2} \bar{p}_{\text {in }}^{2} K^{2} \mu(0)
$$

This clearly shows that the SOA leads to an increase in phase noise variance, with explicit dependence on linewidth enhancement factor and saturation level. Figs. 8 and 9 show good match between (26) and simulation results.

\section{REFERENCES}

[1] D. Zimmerman and L. Spiekman, "Amplifiers for the masses: EDFA, EDWA, and SOA amplets for metro and access applications," J. Lightw. Technol., vol. 22, no. 1, pp. 63-70, Jan. 2004.

[2] B. Mikkelsen, S. Danielsen, C. Joergensen, R. Pedersen, H. Poulsen, and K. Stubkjaer, "All-optical noise reduction capability of interferometric wavelength converters," Electron. Lett., vol. 32, no. 6, pp. 566-567, Mar. 1996.

[3] T. Durhuus, C. Joergensen, B. Mikkelsen, R. Pedersen, and K. Stubkjaer, "All optical wavelength conversion by SOA's in a Mach-Zehnder configuration," IEEE Photon. Technol. Lett., vol. 6, no. 1, pp. 53-55, Jan. 1994.

[4] D. Marcenac, D. Nesset, A. Kelly, and D. Gavrilovic, "40 Gbit/s transmission over $103 \mathrm{~km}$ of NDSF using polarisation independent mid-span spectral inversion by four-wave mixing in a semiconductor optical amplifier," Electron. Lett., vol. 34, no. 1, pp. 100-101, 1998.

[5] A. Ghazisaeidi, F. Vacondio, A. Bononi, and L. A. Rusch, "SOA intensity noise suppression: Multicanonical monte carlo simulator of extremely low BER," J. Lightw. Technol., vol. 27, no. 14, pp. 2667-2677, Jul. 2009.
[6] A. Mecozzi and J. Mork, "Saturation effects in nondegenerate fourwave mixing between short optical pulses in semiconductor laser amplifiers," IEEE J. Sel. Topics Quantum Electron., vol. 3, no. 5, pp. 1190-1207, Oct. 1997.

[7] W. Mathlouthi, P. Lemieux, M. Salsi, A. Vannucci, A. Bononi, and L. Rusch, "Fast and efficient dynamic WDM semiconductor optical amplifier model," J. Lightw. Technol., vol. 24, no. 11, pp. 4353-4365, Nov. 2006

[8] A. Saleh, R. Jopson, and T. Darcie, "Compensation of nonlinearity in semiconductor optical amplifiers," Electron. Lett., vol. 24, no. 15, pp. 950-952, Jul. 1988.

[9] X. Wei, Y. Su, X. Liu, J. Leuthold, and S. Chandrasekhar, "10-Gb/s RZ-DPSK transmitter using a saturated SOA as a power booster and limiting amplifier," IEEE Photon. Technol. Lett., vol. 16, no. 6, pp. 1582-1584, Jun. 2004.

[10] P. Cho, Y. Achiam, G. Levy-Yurista, M. Margalit, Y. Gross, and J. Khurgin, "Investigation of SOA nonlinearities on the amplification of DWDM channels with spectral efficiency up to $2.5 \mathrm{~b} / \mathrm{s} / \mathrm{Hz}$," IEEE Photon. Technol. Lett., vol. 16, no. 3, pp. 918-920, Mar. 2004.

[11] E. Ciaramella, A. D'Errico, and V. Donzella, "Using semiconductor-optical amplifiers with constant envelope WDM signals," IEEE J. Quantum Electron., vol. 44, no. 5, pp. 403-409, May 2008.

[12] J. P. Gordon and L. F. Mollenauer, "Phase noise in photonic communications systems using linear amplifiers," Opt. Lett., vol. 15, no. 23, pp. 1351-1353, Dec. 1990

[13] M. Shtaif, B. Tromborg, and G. Eisenstein, "Noise spectra of semiconductor optical amplifiers: Relation between semiclassical and quantum descriptions," IEEE J. Quantum Electron., vol. 34, no. 5, pp. 869-878, May 1998.

[14] A. Bononi, P. Serena, and A. Orlandini, "A unified design framework for single-channel dispersion-managed terrestrial systems," J. Lightw. Technol., vol. 26, no. 22, pp. 3617-3631, Nov. 2008

[15] K. Kikuchi, C. Zah, and T. Lee, "Measurement and analysis of phase noise generated from semiconductor optical amplifiers," IEEE $J$. Quantum Electron., vol. 27, no. 3, pp. 416-422, Mar. 1991.

[16] X. Wei and L. Zhang, "Analysis of the phase noise in saturated SOAs for DPSK applications," IEEE J. Quantum Electron., vol. 41, no. 4, pp. 554-561, Apr. 2005

[17] G. Agrawal and N. Olsson, "Self-phase modulation and spectral broadening of optical pulses in semiconductor laser amplifiers," IEEE $J$. Quantum Electron., vol. 25, no. 11, pp. 2297-2306, Nov. 1989.

[18] C. Henry, "Theory of the linewidth of semiconductor lasers," IEEE J. Quantum Electron., vol. QE-18, no. 2, pp. 259-264, Feb. 1982.

[19] I. Kang, C. Dorrer, L. Zhang, M. Dinu, M. Rasras, L. Buhl, S. Cabot, A Bhardwaj, X. Liu, M. Cappuzzo, L. Gomez, A. Wong-Foy, Y. Chen, N. Dutta, S. Patel, D. Neilson, C. Giles, A. Piccirilli, and J. Jaques, "Characterization of the dynamical processes in all-optical signal processing using semiconductor optical amplifiers," IEEE J. Sel. Topics Quantum Electron., vol. 14, no. 3, pp. 758-769, Mat/Jun. 2008.

[20] J. Hansryd, J. van Howe, and C. Xu, "Experimental demonstration of nonlinear phase jitter compensation in DPSK modulated fiber links," IEEE Photon. Technol. Lett., vol. 17, no. 1, pp. 232-234, Jan. 2005.

[21] K. Ho and J. Kahn, "Electronic compensation technique to mitigate nonlinear phase noise," J. Lightw. Technol., vol. 22, no. 3, pp. 779-783, Mar. 2004.

[22] J. van Howe, G. Zhu, and C. Xu, "Compensation of self-phase modulation in fiber-based chirped-pulse amplification systems," Opt. Lett., vol. 31, no. 11, pp. 1756-1758, Jun. 2006.

[23] G. Zhu, L. Mollenauer, and C. Xu, "Experimental demonstration of postnonlinearity compensation in a multispan DPSK transmission," IEEE Photon. Technol. Lett., vol. 18, no. 9, pp. 1007-1009, May 2006.

[24] A. Lau and J. Kahn, "Signal design and detection in presence of nonlinear phase noise," J. Lightw. Technol., vol. 25, no. 10, pp. 3008-3016, Oct. 2007.

[25] C. Xu and X. Liu, "Postnonlinearity compensation with data-driven phase modulators in phase-shift keying transmission," Opt. Lett., vol. 27 , no. 18 , pp. 1619-1621, 2002

[26] A. Saleh and I. Habbab, "Effects of semiconductor-optical-amplifier nonlinearity on the performance of high-speed intensity-modulation lightwave systems," IEEE Trans. Commun., vol. 38, no. 6, pp. 839-846, Jun. 1990. 
[27] A. Bhardwaj, C. Doerr, S. Chandrasekhar, and L. Stulz, "Reduction of nonlinear distortion from a semiconductor optical amplifier using an optical equalizer," IEEE Photon. Technol. Lett., vol. 16, no. 3, pp. 921-923, Mar. 2004.

[28] J. Downie and J. Hurley, "Performance of an MLSE-EDC receiver with SOA-induced nonlinear impairments," IEEE Photon. Technol. Lett., vol. 20, no. 15, pp. 1326-1328, Aug. 2008.

[29] X. Li and G. Li, "Electrical postcompensation of SOA impairments for fiber-optic transmission," IEEE Photon. Technol. Lett., vol. 21, no. 9, pp. 581-583, May 2009.

[30] I. T. Monroy, F. Öhman, K. Yvind, L. J. Christiansen, J. Mörk, C. Peucheret, and P. Jeppesen, "Monolithically integrated reflective SOA-EA carrier re-modulator for broadband access nodes," Opt. Exp., vol. 14, no. 18, pp. 8060-8064, 2006.

[31] M. Kroh, G. Unterborsch, G. Tsianos, R. Ziegler, A. Steffan, H. Bach, J. Kreissl, R. Kunkel, G. Mekonnen, W. Rehbein, D. Schmidt, R. Ludwig, K. Petermann, J. Bruns, T. Mitze, K. Voigt, and L. Zimmermann, "Hybrid integrated $40 \mathrm{gb} / \mathrm{s}$ DPSK receiver on SOI," in Proc. Conf. Opt. Fiber Commun. 2009 (OFC 2009), , pp. 1-3-.

[32] J. M. Hutchinson, J. S. Barton, M. L. Masanovic, M. N. Sysak, J. A. Henness, L. A. Johansson, D. J. Blumenthal, and L. A. Coldren, M. Osinski, H. Amano, and F. Henneberger, Eds., "Monolithically integrated InP-based tunable wavelength conversion," Proc. SPIE vol. 5349, no. 1, pp. 176-184, 2004 [Online]. Available: http://link.aip.org/link/?PSI/ 5349/176/1, [Online]. Available

[33] J. Leuthold, C. Joyner, B. Mikkelsen, G. Raybon, J. Pleumeekers, B. Miller, K. Dreyer, and C. Burrus, "100 gbit/s all-optical wavelength conversion with integrated SOA delayed-interference configuration," Electron. Lett., vol. 36, no. 13, pp. 1129-1130, Jun. 2000.

[34] A. Saleh, "Nonlinear models of travelling-wave optical amplifiers," Electron. Lett., vol. 24, no. 14, pp. 835-837, Jul. 1988.

[35] D. Cassioli, S. Scotti, and A. Mecozzi, "A time-domain computer simulator of the nonlinear response of semiconductor optical amplifiers," IEEE J. Quantum Electron., vol. 36, no. 9, pp. 1072-1080, Sep. 2000.

[36] A. Ghazisaeidi, F. Vacondio, A. Bononi, and L. A. Rusch, "Statistical characterization of bit patterning in SOAs: BER prediction and experimental validation," presented at the Conf. Opt. Fiber Commun. 2009 (OFC 2009), San Diego, CA, Paper OWE7.

[37] A. Ghazisaeidi, F. Vacondio, A. Bononi, and L. A. Rusch, "Bit patterning in SOAs: Statistical characterization through multicanonical monte carlo simulations," IEEE J. Quantum Electron., to be published.

[38] X. Wei, X. Liu, and C. Xu, "Numerical simulation of the SPM penalty in a 10-Gb/s RZ-DPSK system," IEEE Photon. Technol. Lett., vol. 15, no. 11, pp. 1636-1638, Nov. 2003.

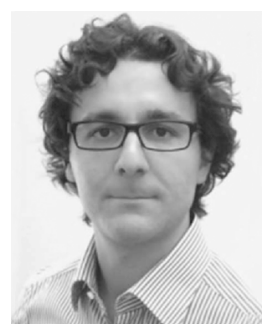

Francesco Vacondio was born in Reggio Emilia, Italy, on December 18, 1981. He received the Laurea Magistrale degree (cum laude) in electrical engineering from the University of Parma, Parma, Italy. He is currently working toward the Ph.D. degree in the Department of Electrical and Computer Engineering and the Center for Optics, Photonics and Lasers, Université Laval, Qubec City, Canada.

His current research interests include performance analysis and design of spectrally efficient optical networks, the exploitation of nonlinear properties of semiconductor optical amplifiers, and microwave photonics.

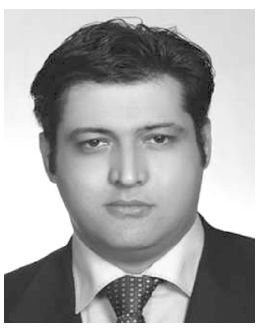

Amirhossein Ghazisaeidi received the B.S. degree in electrical engineering and the M.A. degree in communications systems from Sharif University of Technology, Tehran, Iran. He is currently working toward the Ph.D. degree in the Department of Electrical and Computer Engineering and the Center for Optics, Photonics and Lasers, Université Laval, Quebec City, Canada.

His current research interests include optical-code-division multiple access and spectrum-sliced wavelength-division multiplexing using incoherent sources, dynamics, and noise properties of optical amplifiers, modeling optoelectronic devices, and performance analysis of optical links.

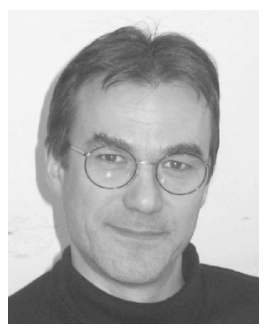

Alberto Bononi received the Laurea degree (cum laude) in electronics engineering from the University of Pisa, Pisa, Italy, in 1988, and the M.A. and $\mathrm{Ph} . \mathrm{D}$. degrees in electrical engineering from Princeton University, Princeton, NJ, 1992 and 1994, respectively.

During 1990, he was with GEC-Marconi Hirst Research Centre, Wembley, U.K., on a Marconi S.p.A. project on coherent optical systems. From 1994 to 1996, he was an Assistant Professor in the Electrical and Computer Engineering Department, State University of New York, Buffalo, where he teaches courses in electric circuits and optical networks. During 1997 and 1999, he was a Visiting Faculty in the Département de Genie Électrique, Université Laval, QC, Canada, where he was engaged in research on fiber amplifiers. Currently, he is an Associate Professor of telecommunications in the Dipartimento di Ingegneria dell'Informazione, School of Engineering, Università degli Studi di Parma, Parma, Italy, where he teaches courses in probability theory and stochastic processes, telecommunications networks, and optical communications. His current research interests include system design and performance analysis of high-speed all-optical networks, nonlinear fiber transmission for wavelength-division multiplexing systems, linear and nonlinear polarization-mode dispersion, and transient gain dynamics in optical amplifiers.

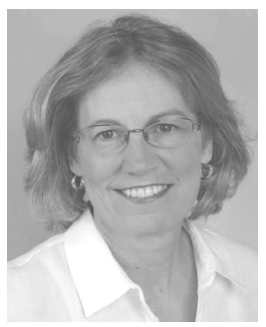

Leslie Ann Rusch (S'91-M'94-SM'00) received the B.S.E.E. (honors) degree from California Institute of Technology, Pasadena, in 1980, and the M.A. and $\mathrm{Ph} . \mathrm{D}$. degrees in electrical engineering from Princeton University, Princeton, NJ, in 1992 and 1994, respectively.

From 2001 to 2002, she was the Manager of a group researching new wireless technologies at Intel Corporation. She is currently a Full Professor in the Department of Electrical and Computer Engineering and the Center for Optics, Photonics and Lasers, Université Laval, Qubec City, Canada, where she is engaged in research in wireless and optical communications. Her research interests include optical-code-division multiple access and spectrum-sliced wavelength-division multiplexing using incoherent sources for passive optical networks, semiconductor and erbium-doped optical amplifiers and their dynamics, radio over fiber, and in wireless communications, high performance, and reduced complexity receivers for ultra-wideband systems employing optical processing. 